\title{
Early fire sensing using near-IR diode laser spectroscopy
}

\author{
David S. Bomse, D. Christian Hovde, Shin-Juh Chen, and Joel A. Silver \\ Southwest Sciences, Inc.
}

\begin{abstract}
We describe research leading to a trace gas detection system based on optical absorption using near-IR diode lasers that is intended to provide early warning of incipient fires. Applications include "high loss" structures such as office buildings, hospitals, hotels and shopping malls as well as airplanes and manned spacecraft where convention smoke detectors generate unacceptably high false alarm rates. Simultaneous or near-simultaneous detection of several gases (typically carbon dioxide, carbon monoxide, acetylene and hydrogen cyanide) provides high sensitivity while reducing the chance of false alarms. Continuous measurement of carbon dioxide concentrations also provides an internal check of instrument performance because ambient levels will not drop below $\sim 350 \mathrm{ppm}$.
\end{abstract}

key words: fire sensing, spectroscopy, laser, trace gas

\section{INTRODUCTION}

Early fire sensing by detecting fire-related gases can improve response times and minimize false alarms for high loss structures such as office buildings, hotels, shopping malls, and hospitals, and for aircraft and manned spacecraft. In theory, the relative concentrations of the target gases can provide information about the type and extent of the fire. In practice, this is a difficult gas sensing problem. Gases from incipient (i.e., smoldering) fires are usually diluted by surrounding air area prior to detection; high sensitivity measurements are needed. The most common fire products - carbon dioxide, carbon monoxide, and water vapor - usually have large background concentrations; it can be difficult to assign changes in concentration to fire instead of to other likely sources. Monitoring large areas, such as office buildings, requires numerous sensors or a distributed sampling system; this can be expensive.

We describe fire detection using optical absorption spectroscopy with near-infrared diode lasers for (nearly) simultaneous quantification of three or more target gases. We use the type of lasers developed for high speed, trunk line, fiber optic communications. These commercial devices operate near room temperature, are fabricated with distributed feedback (DFB) structures to guarantee single mode operation, and are compatible with inexpensive InGaAs photodiode detectors that also operate at room temperature. These "user friendly" characteristics outweigh the loss of sensitivity inherent in near-infrared spectroscopic measurements accessing overtone vibrations that are, typically, one to three orders of magnitude weaker than the fundamental, mid-infrared transitions.

Much of the results described in this manuscript comes from two recently completed projects demonstrating the diode laser approach for incipient fires. We emphasized simultaneous detection of multiple gases and the ability to distribute the light from one set of diode lasers - using one laser per gas - to several hundred measurement locations using fiber optics. Ongoing work at Southwest Sciences is aimed at fire sensing in aircraft fire compartments and on board manned spacecraft.

\subsection{Which gases?}

Ideally, the gases useful for fire sensing would be ubiquitous to incipient fires; have relatively large near-infrared absorption cross sections; exhibit sharp, isolated absorption lines free of interference from common background gases such as water vapor; show low background concentrations in the absence of fire; and, be chemically stable enough to survive transport from the fire source to the measurement location. No species meet all of these criteria. The gases generated in the largest

*dbomse@swsciences.com; phone 1505 984-1322; fax 1505 988-9230; http://www.swsciences.com; 1570 Pacheco Street; Suite E-11; Santa Fe, NM, USA 87505 
quantities by most fires (carbon dioxide, and water vapor) have large atmospheric backgrounds. Carbon monoxide, which is most characteristic of smoldering fires, can also show large, fluctuating background concentrations, and has relatively small near-infrared absorption features.

Fourier transform infrared (FTIR) measurements of a mix of commercial plastics has been used by the FAA to determine gas phase products likely in aircraft cargo fires. ${ }^{1}$ Nonflaming pyrolysis produces significant amounts of carbon monoxide, carbon dioxide, fluorophosgene, hydrogen chloride, hydrogren cyanide, sulfur dioxide, methane, ethylene, and water vapor. The same material undergoing open flame combustion emits $\mathrm{CO}, \mathrm{CO}_{2}, \mathrm{HCl}, \mathrm{HCN}, \mathrm{NO}, \mathrm{CH}_{4}$, acetylene, ethylene, and water vapor. Table I lists the gases that can be detected readily using near-infrared diode laser spectroscopy, the expected detection limits, and detection wavelength. Sensitivities are given in column density units (ppm$\mathrm{m})$ and correspond to a minimum detectable absorbance of $1 \times 10^{-5}$. Carbon monoxide, $\mathrm{CO}_{2}, \mathrm{HCN}$, and acetylene are accessible using readily available communications lasers operating between 1520 and $1580 \mathrm{~nm}$. Methane, ethylene, and $\mathrm{HCl}$ require wavelengths longer than $1600 \mathrm{~nm}$. Communicationsstyle lasers can be fabricated for operation at those wavelengths, but are not routinely available. This narrows the target group down to $\mathrm{CO}, \mathrm{CO}_{2}, \mathrm{HCN}$, and acetylene. We include $\mathrm{CO}$ despite its relatively small absorption cross section because it is generated by nearly all types of combustion.

Carbon dioxide is included for a different reason. Its presence is virtually guaranteed under all conditions. As such, it can be used as an internal confirmation of instrument operation and, thereby, eliminating the need for frequent calibration. The remaining two

Table I- Detection limits and near-infrared laser wavelengths for combustion-related gases

\begin{tabular}{|l|c|l|}
\hline Gas & $\begin{array}{l}\text { Sensitivity } \\
(\mathbf{p p m}-\mathbf{m})^{\mathbf{a}}\end{array}$ & $\begin{array}{l}\text { Wavelength } \\
(\mathbf{n m})\end{array}$ \\
\hline carbon dioxide $\left(\mathrm{CO}_{2}\right)$ & 30 & 1570 \\
\hline carbon monoxide $(\mathrm{CO})$ & 30 & 1570 \\
\hline methane $\left(\mathrm{CH}_{4}\right)$ & 0.6 & 1650 \\
\hline acetylene $(\mathrm{HCCH})$ & 0.08 & 1529 \\
\hline ethylene $\left(\mathrm{H}_{2} \mathrm{CCH}_{2}\right)$ & $\mathrm{b}$ & $1610-1630$ \\
\hline hydrogen cyanide $(\mathrm{HCN})$ & 0.08 & 1529 \\
\hline hydrogen chloride $(\mathrm{HCl})$ & 0.15 & 1790 \\
\hline
\end{tabular}

a. Assumes absorbance sensitivity of $1 \times 10^{-5}$

b. FTIR shows near-IR band position, but not absolute sensitivity gases are particularly useful for fire sensing because they can be detected at sub-ppm concentrations and, in most environments, have near zero background concentrations. Hydrogen cyanide is generated by combustion or pyrolysis of the type of nitrogen-containing plastics (such as urethane foams) commonly used in furniture, aircraft interiors, and rigid polymers. Acetylene is a key soot precursor and is generated by pyrolysis of nearly all organic compounds.

\subsection{Wavelength Modulation Spectroscopy (WMS)}

Wavelength modulation spectroscopy is described in detail elsewhere. ${ }^{2-3}$ Briefly, it is a useful trace gas detection technique because it shifts the detection band from frequencies near DC, where laser output is characterized by excess noise (i.e., $1 / f$ noise) to high frequencies where excess noise is unimportant. Laboratory measurements have achieved minimum detectable absorbances approaching the shot noise limit $\left(\sim 10^{-8}\right.$ for near-infrared lasers operating at several $\left.\mathrm{mW}\right)$ but, in practice, unwanted optical interference fringes - etalons - constrain detection limits to $10^{-5}$ fractional absorbance. This is adequate to achieve ppm detection limits for many gases, Table I.

Wavelength modulation is accomplished by adding an AC component to the laser drive current. This is effective because diode lasers tune with current. Absorption by the target gas converts the wavelength modulation (which is, strictly speaking, a frequency modulation) to an amplitude modulation and introduces AC components to the output of the photodetector. Those components occur at the modulation frequencies and its integral harmonics. One of the components is selected for processing using a demodulator such as a mixer or lock-in amplifier. We usually select the $2 f$ component because the processed signals are zero in the absence of absorption (ideally) and the WMS signal peaks at the center of the absorption line. The resulting spectrum looks similar to the second derivative of the absorption line. For the work described here, the demodulation was performed numerically following digitization of the photodetector output. 


\section{EXPERIMENTAL}

We describe two applications of wavelength modulation spectroscopy for detecting trace gases for fire detection. The first system, constructed for the U.S. Department of Commerce (DOC), allows simultaneous detection of carbon dioxide, carbon monoxide, and hydrogen cyanide. The other - a demonstration project for NASA - used one distributed feedback (DFB) communications laser to measure hydrogen cyanide and acetylene. Both projects relied on fiber optics to deliver the laser light to a multiple pass, Herriott-style measurement cell. ${ }^{4}$

The Department of Commerce (DOC) instrument is novel for two reasons. First, several gases were detected simultaneously using one optical path and one detector by combining the output from three diode lasers. The wavelength of each laser is modulated at a different frequency. ${ }^{5}$ The photodiode output contains information specific to the power and absorbance for each of the lasers (gases). This approach is similar to television or radio broadcasting where separate information is transmitted using closely spaced carrier frequencies. The advantages of this modulation frequency multiplexing approach are a simplification of the measurement cell optics - all three beams traverse the identical path and a simplification of the detector electronics because only one photodetector is needed instead of three.

The second novel feature was the use of digital signal processing (DSP) electronics for laser control and signal processing. This made it possible to develop a relatively compact prototype instrument that contained three laser systems, their control electronics, line locking reference cells, sophisticate data processing, and serial communications to a host, personal computer. Although we started working with DSP systems because their instruction sets are optimized for the types of operations needed to implement wavelength modulation spectroscopy (numerical demodulation, filtering, and matrix operations), we soon realized that the diode laser applications are not terribly demanding computationally. Most modern microprocessors are adequate for real time measurements and analysis. Instead, we soon realized that the real benefit of DSP microcontrollers is their simple interfacing to high performance digital-to-analog and analog-to-digital converters. Complete systems are more compact and less expensive than single board computers based on conventional microprocessors.

The laser wavelength is modulated and ramped simultaneously and the demodulated signals are full WMS spectra. Figure 1 illustrates a key portion of the DSP program. Direct memory access (DMA) controllers output a user-defined number of periods of the eight-element sinusoidal modulation waveform. (The default value is eight periods.) Analog electronic components combine that modulation waveform with the current value of the laser sweep waveform. At the same time, the preamplified and bandpassed signal from the Herriott multiple pass cell detector is being digitized and stored using a second DMA channel. Double buffering allows continuous operation; one data set is being processed (demodulated) while another is being collected. The figure represents the double buffering by showing the signal from the Herriott cell entering the left hand buffer while the data from the right hand buffer is applied to the $2 f$ demodulation routine. The demodulated data form one point in a WM

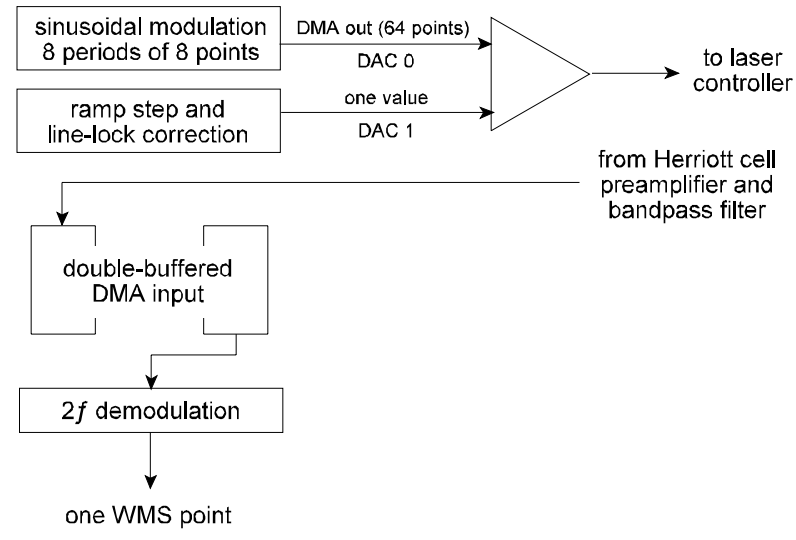

Figure 1 - Component of DSP program showing synchronized input and output signals. spectrum corresponding to one step in the sweep ramp.

The software demodulation in the DSP program entails first shifting the data array to account for the synchronization delay between the input and output DMA data streams. A Welch window is then applied to the data. This function goes to zero at the end points and is quadratic in between reaching a maximum of one at the midpoint. The window reduces the harmonic content of the data. The actual demodulation includes two vector dot products. The windowed data are dotted 
with the $2 f$ sine function and with the $2 f$ cosine function. The resulting scalars are then weighted by the sin and cosine of the demodulation phase, respectively, and summed. This process gives much finer phase control than is possible using a phased demodulation waveform. The process is repeated for all points within the sweep ramp to generate a full wavelength demodulation spectrum. Data from several (typically eight) ramps are co-added to create a one second measurement. A similar set of demodulations and co-adds creates the corresponding $1 f$ spectrum that is used as a measurement of the laser intensity.

In our data analysis method, an experimentally acquired spectrum from the Herriott cell is characterized as the sum of the "true" WMS line shape, an offset, a linear baseline slope, and some baseline curvature. The "true" line shape is an experimentally acquired spectrum at relatively large gas concentrations. A multilinear least-squares fit determines the contributions of each term to a raw spectrum. The fitting coefficient corresponding to the WMS line shape is proportional to the target gas concentration. This data analysis method is shown pictorially in Fig. 2 and is implemented in a matrix formalism. We construct a $4 \times$ $\mathrm{N}$ reference matrix where $\mathrm{N}$ is the number of points in each spectrum. The four columns consist of the "true" WMS spectrum and the three baseline components (offset, slope, and curvature). The reference matrix is "inverted" ahead of time so that the final calculation is extremely fast. In practice, we care only about the contribution from the "true" $2 f$ component. The computation is reduced to a single vector, dot-product using the experimental $2 f$ spectrum and the first column of the "inverted" matrix.

The modulation frequency multiplexing method used in the DOC instrument allows simultaneous measurements of three gases. The light output from each laser is combined using fiber optics. As a result, all three beams are guaranteed to traverse the same optical path through the Herriott cell and only one detector is needed. Each laser is wavelength modulated at a different frequency much as signals from different radio and television stations are transmitted using different carrier frequencies. Effective implementation of the approach requires demodulating the detector output to recover six separate signals: the $1 f$ and $2 f$ waveforms for each laser. These demodulations are performed using the DSP microcontrollers.

Figure 3 shows the observed variation is signal rejection as a function of frequency for one of the systems. The DSP microcontroller was configured to demodulate an incoming data stream at $20 \mathrm{kHz}$ and report the rms magnitude of the demodulated signal for detection conditions similar to those used during gas detection. The test inputs were sine waves from a commercial function generator. The data show that adding a Welch window to the digitized data before demodulation and low-pass filtering the spectra after improves signal

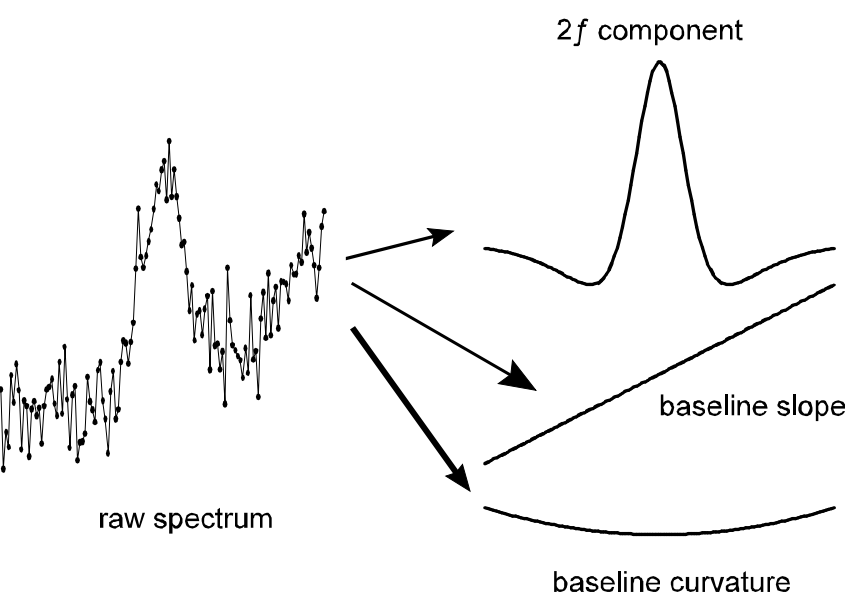

Figure 2 - Decomposition of raw spectrum into $2 f$ component plus the baseline slope and curvature. (The baseline offset term is not included.)

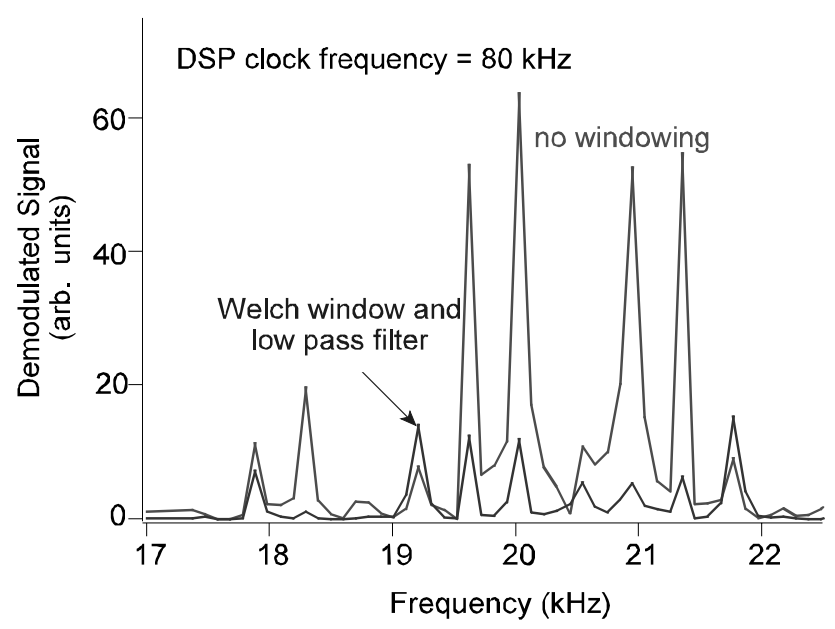

Figure 3 - RMS demodulated signals as a function of input frequency for DSP demodulation at $20 \mathrm{kHz}$. The Welch windowed and low pass filtered data best simulate the gas analyzer operation. 
rejection significantly. Windowing data is a standard digital signal processing technique that reduces the harmonic content of the raw data and is particularly when working with relatively small data sets. Data acquired over short periods of time may appear to include contributions at numerous frequencies because only a few periods of the base frequency have been sampled. As a result, the demodulation pass band does not vary smooth with frequency and it is important to select carefully the modulation and demodulation frequencies for the three DSP systems. Data such as those shown in Fig. 3 allowed us to pick $1 f$ frequencies of $9.10,10.0$, and $10.95 \mathrm{kHz}$ for $\mathrm{CO}_{2}, \mathrm{CO}$, and $\mathrm{HCN}$, respectively.

The demonstration instrument built for the NASA project used only one laser operating near $1529 \mathrm{~nm}$ to detect $\mathrm{HCN}$ and acetylene. Absorption lines of comparable line strength for the two gases lie within $0.3 \mathrm{~cm}^{-1}$ of each other, and can both be measured by sweeping the laser current at fixed laser temperature. The instrument was similar to the DOC device in that digital signal processing methods were used to control the laser, apply the modulation waveform, digitize the photoreceiver output, perform and numerical demodulation, and extract gas concentrations from the $2 f$ spectra. The big differences between the two approaches are 1) the NASA instrument added a noise canceler circuit to remove the common mode signals introduced by the laser distortions and fiber optics, ${ }^{6}$ and 2) the DC portion of the photodiode current was measured directly. Since only one laser was used, there was no need to rely on the $1 f$ component of the photoreceiver output to determine the light intensity.

The noise canceler approach uses a simple transistor-pair current divider to eliminate common mode signals between two optical paths. ${ }^{6}$ In our case, the first path is obtained by splitting off a portion of the light beam as it enters the multiple pass cell while the second path is the beam that has traversed the cell. Since second harmonic amplitude distortion is common to both signals, it is eliminated and the resulting WMS spectra show significantly flatter baselines and the WMS signals are much more prominent. This improvement is significant for development of the DSP-based instrument because it reduces the dynamic range required to acquire the WMS spectra. This allows us to work with signal levels that are wellmatched to the performance of the analog-to-digital converters without needing software-controlled, variable gain amplifiers.

\section{RESULTS and DISCUSSION}

\subsection{Gas Detection Sensitivities}

Figure 4 shows calibration curves for the three gases $\mathrm{CO}, \mathrm{CO}_{2}$, and $\mathrm{HCN}$ - obtained from the DOC instrument. Carbon dioxide $\left(\mathrm{CO}_{2}\right)$ is detected using the $\mathrm{R}(16)$ line at $6359.97 \mathrm{~cm}^{-1}$. The atmospheric pressure, room temperature absorption cross section is $8.1 \times 10^{-23}$

$\mathrm{cm}^{2}{ }^{7}$ Calibration data show a linear response up to the largest concentration tested, $1750 \mathrm{ppm}$. Standard deviations for the data points average $36 \mathrm{ppm}$; they range from 32 to $51 \mathrm{ppm}$ with all but two of the values lying below $40 \mathrm{ppm}$. The average error corresponds to a minimum detectable absorbance of $6.2 \times 10^{-5}$ and is limited by etalons within the fiber optics.

These results verify one of the key expectations of the diode-laser-based fire detection method: continuous measurement of carbon dioxide concentrations can be used as an automatic, internal check of instrument performance. $\mathrm{CO}_{2}$ background concentrations - about

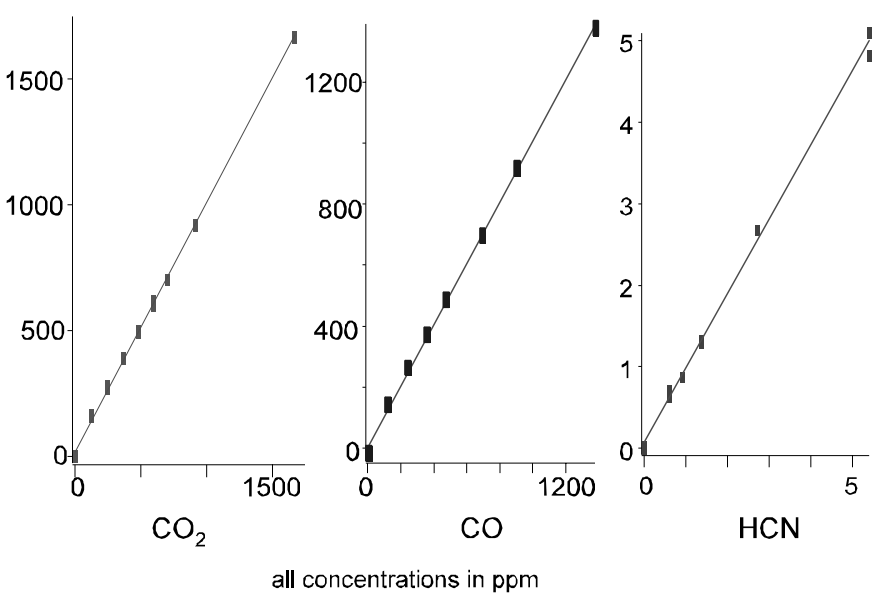

Figure 4 - Calibration curves for the three gases detected by the DOC fire sensing instrument. $350 \mathrm{ppm}$ - can be measured with a signal-to-noise ratio of 10 which is more than adequate for confirming instrument performance. 
Carbon monoxide (CO) is detected using the $\mathrm{R}(14)$ line at $6395.43 \mathrm{~cm}^{-1}$. The atmospheric pressure, room temperature absorption cross section is $6.5 \times 10^{-23} \mathrm{~cm}^{2}{ }^{7}$ A good indication of the small signal performance is obtained by examining the raw data for a set of $\mathrm{CO}$ concentrations between 0 and $47 \mathrm{ppm}$, Fig. 5. These data reveal the slow baseline drift associated with variations in an etalon present in the laser's fiber optics. This etalon occurs somewhere within the diode laser fiber output train and is impervious to known etalon-reduction techniques (although some improvement is possible from using index matching gel in the fiber output connector). The data in Fig. 10 imply that short term concentration changes $>25 \mathrm{ppm}$ can be measured reliably, while smaller changes are obscured by baseline drift for time periods on the order of 15 minutes. The combination of the $\mathrm{CO}$ absorption cross section obtained from the HITRAN data base ${ }^{7}$ and the $9.06 \mathrm{~m}$ Herriott cell path length imply a minimum detectable absorbance (corresponding to $25 \mathrm{ppm} \mathrm{CO}$ ) of $3.6 \times 10^{-5}$.

Hydrogen cyanide is detected using the $\mathrm{R}(6)$ line at $6539.12 \mathrm{~cm}^{-1} .8$ Standard deviations for the individual data points range from 0.098 to $0.135 \mathrm{ppm}$ and average $0.11 \mathrm{ppm}$. The average value corresponds to an absorbance noise of $6.6 \times 10^{-5}$ assuming the calculated cross section.

\subsection{Cross talk and laser power dependence}

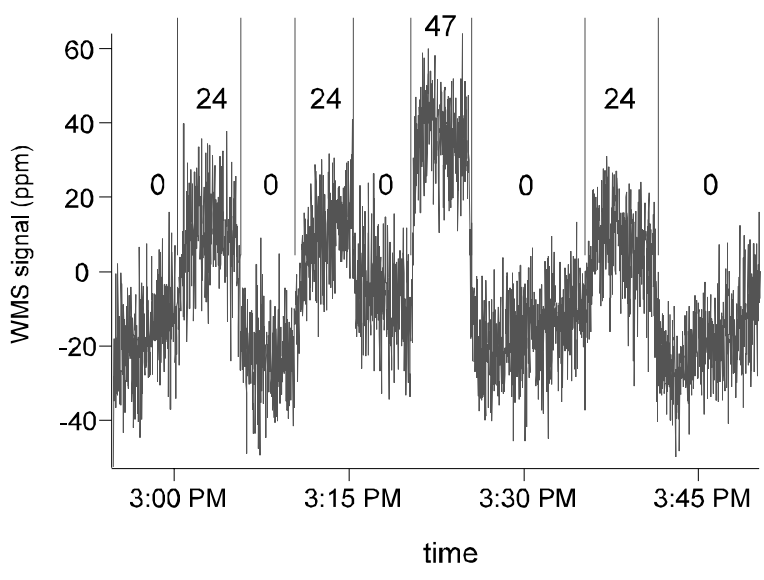

Figure 5 - Diode laser instrument response to small concentrations of $\mathrm{CO}$.

Useful operation of the DOC instrument requires eliminating cross talk among the three detection channels. In other words, large amounts of one gas should not introduce false signals in either of the other two channels. Data in Fig. 6 show that no cross talk is measured despite large changes in gas concentrations. This experiment used only $\mathrm{CO}$ and $\mathrm{CO}_{2}$ because the gases are easier to handle in large concentrations than is $\mathrm{HCN}$.

The design specification for the DOC instrument (and for all other diode-laser-based sensors that we have put in the field) is for a minimum detectable absorbance of $1 \times 10^{-5}$. Unwanted optical interference fringes (etalons) are usually the dominant source of measurement uncertainty and overwhelm fundamental noise sources due to laser/detector shot noise and detector thermal noise. As a result, a 2 $\mathrm{mW}$ laser beam could be distributed over 830 measurement locations and there would be sufficient optical power at each measurement point that the fundamental noise sources would be an order of magnitude smaller than the target minimum detectable absorbance of $1 \times 10^{-5}$. Figure 7 shows simultaneous measurements of $\mathrm{CO}$ and $\mathrm{CO}_{2}$ for laser powers of 179,18 , and $1.4 \mu \mathrm{W}$. The detection sensitivity was nearly invariant, although the lowest powers showed more high frequency noise. The

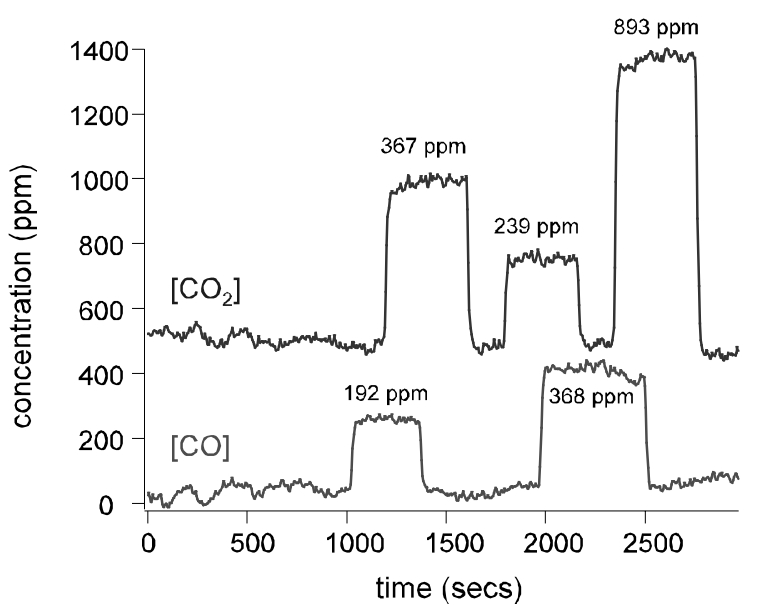

Figure 6 - Cross talk test between $\mathrm{CO}$ and $\mathrm{CO}_{2}$ detection channels. measurements were obtained using the $1 \times 8$ fiber optic beam splitters. Putting one in line reduced the power throughput by an order of magnitude (a factor of 8 plus insertion losses), while adding a second knocked down the transmitted power by another factor of 13 . 
The latest generation of commercial, near-infrared diode lasers have minimum power specifications of $20 \mathrm{~mW}$. Distribution to over 1000 sites is clearly possible.

\subsection{Combining the Noise Canceler with WMS}

Figure 8 shows wavelength modulation spectra acquired with the NASA instrument prior to adding the noise canceler circuitry. The traces, from measurements of 2.7 ppm $\mathrm{HCN}$ and of pure $\mathrm{N}_{2}$, reveal a potential limitation to the usefulness of gas detection using near-infrared lasers. The prominent, upward-curving baselines are due to second harmonic distortion in the amplitude modulation of the laser. In other words, the $10 \mathrm{kHz}$ sinusoidal modulation waveform applied to the laser drive current induces both synchronous amplitude and wavelength modulation. It also creates a small amount of amplitude modulation at $20 \mathrm{kHz}$ (second harmonic distortion of the

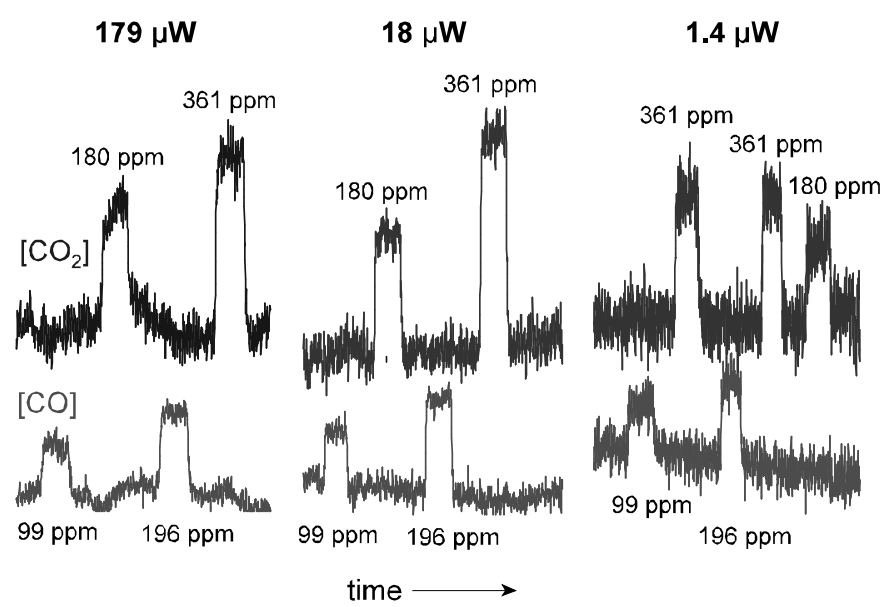

Figure 7 - Simultaneous detection of $\mathrm{CO}$ and $\mathrm{CO}_{2}$ over a 128 -fold reduction in laser power. AM) that is indistinguishable from the true WMS signal. The largest part of the demodulated distortion-induced signal, at the high frequency end of the scan, corresponds to an absorbance of $1 \times 10^{-3}$ which is 100 times the target minimum detectable absorbance for the Phase I instrument.

Adding "noise canceler" circuitry to the Herriott cell photoreceiver resolved the problem, Fig 9. The "lumpiness" of the baseline in the lower spectrum is due to unwanted optical interference fringes (etalons) caused by vignetting of the beams on the noise canceler detectors. We used a commercial noise canceler containing built-in, small diameter photodiodes. The optical beams in our system overfilled the diodes, resulting in the etalons seen. Based on the mismatch between the beam size and detector diameters, we anticipate a factor of four reduction in baseline uncertainty is possible using optimized photodiodes.

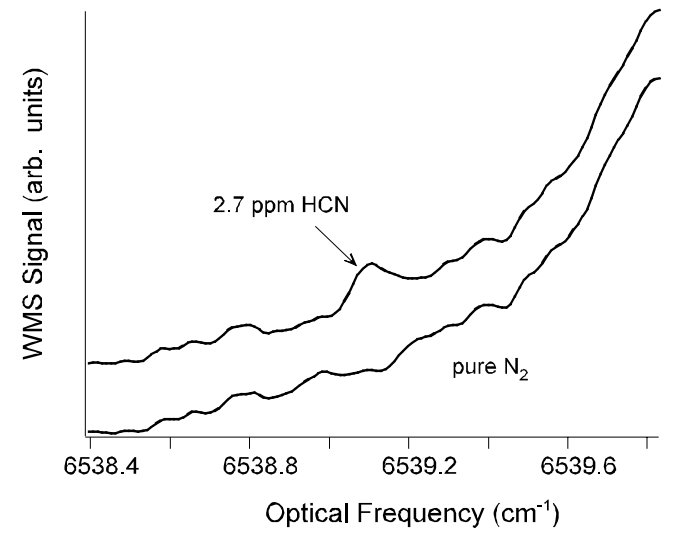

Figure 8 - Wavelength modulation spectra of 2.7 ppm HCN and of $\mathrm{N}_{2}$ baseline showing the effects of second harmonic AM distortion.

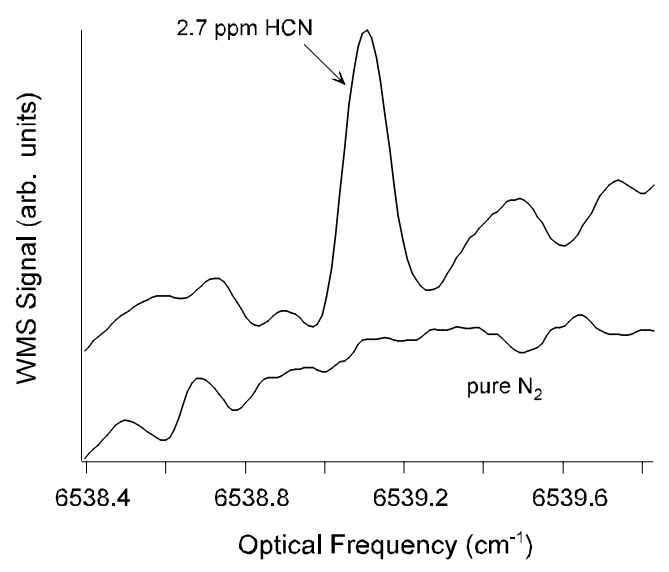

Figure 9 - WMS spectra obtained using the noise canceler photoreceiver to remove laser second harmonic distortion. 


\subsection{Hydrogen cyanide and acetylene from burning upholstery foam}

The NASA instrument was used successfully to detect hydrogen cyanide and acetylene generated from combustion of a small $(125 \mathrm{mg})$ piece of upholstery foam. The results are shown in Fig. 10 with the acetylene data displaced vertically for clarity. The multiple pass cell was placed on the counter top within a fume hood. Spectra such were collected at $5 \mathrm{~Hz}$ and analyzed to extract the contributions due to the two gases. The upholstery foam, in an aluminum weighing dish placed about $10 \mathrm{~cm}$ from the center of the cell, was ignited using a butane lighter 29 seconds into the experiment. The flame selfextinguished about eight seconds later and the lighter was used to re-ignite the foam at the 38 second mark. It burned steadily for another 15 seconds, leaving a black residue that smoldered until the sample was removed at the 76 second mark.

Acetylene dominates gas production when open flame is present, then the two gases correlate well after about the 40 second mark when the foam residue was smoldering. Even though the sample was placed fairly close to the measurement point, the emitted gases were diluted quickly by the fume hood air flow and the location of the Herriott cell - on the hood counter top instead of higher up - also reduced the effectiveness of the gas sampling. Nevertheless, the high sensitivity and rapid response time of the diode laser method allowed unambiguous measurements of the two gases at concentrations well below $1 \mathrm{ppm}$. These results clearly demonstrate the usefulness of the diode-laser method for detecting combustion products.

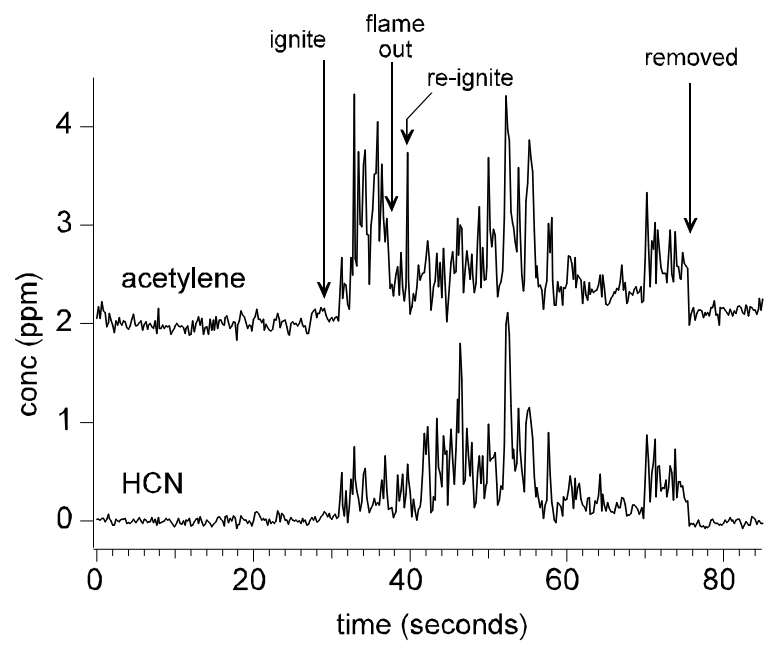

Figure 10 - Direct, real-time $(5 \mathrm{~Hz})$ measurements of $\mathrm{HCN}$ and acetylene from combustion of $125 \mathrm{mg}$ of upholstery foam. The acetylene data have been displaced vertically by 2 ppm for clarity.

\section{CONCLUSIONS}

Wavelength modulation spectroscopy with diode lasers is an effective way to detect trace gases generated by fires and, as such, should be useful for early detection of fires. Simultaneous or near-simultaneous measurement of multiple species should reduce false alarm rates. Response times are limited by gas transport to the measurement location and not by the spectroscopic technique. For example, target specifications for the next generation of fire sensors within aircraft cargo compartments call for overall response times of one minute or less. Assuming thirty seconds for gas to circulate to the measurement point leaves half a minute for the actual measurement. This is well within the time scales demonstrated by the DOC and NASA instruments.

Digital signal processing (DSP) hardware and software are useful for constructing compact, reliable diode-laser-based sensors. Although simultaneous detection of multiple gases is possible using modulation frequency multiplexing methods (as was done with DOC instrument), the improved sensitivity from combining the noise canceler with wavelength modulation obviates the advantages of the simultaneous measurements. Sequential detection gives better sensitivity. Fiber optics make it possible to distribute the light from one laser (or set of lasers) to as many as one thousand measurement locations. This is particularly useful when considering fire detection in high loss structures such as office buildings, hotels, hospitals, and shopping malls.

Ongoing work will determine the true effectiveness of multiple gas detection for reducing false alarm rates. The suite of gases being studied - $\mathrm{CO}, \mathrm{CO}_{2}, \mathrm{HCN}$, and acetylene - provide a good mix of generality and sensitivity using readily available diode lasers. Additional gases such as hydrogen chloride, ethylene, and methane would also be useful for fire detection, but require longer wavelength lasers that are not routinely available. 


\section{ACKNOWLEDGMENTS}

Southwest Sciences gratefully acknowledges support from the U.S. Department of Commerce through contract number 50DKNB-7-90149 from NASA through contracts number NAS9-01031 and NAS9-02020.

\section{REFERENCES}

1. L. C. Speitel, Fourier transform infrared analysis of combustion gases," FAA Report Number DOT/FAA/AR-01/88 (October, 2001).

2. J. A. Silver, Appl. Opt. 31, 707 (1992).

3. D. S. Bomse, A. C. Stanton, and J. A. Silver, Appl. Opt. 31, 718 (1992).

4. D. R. Herriott, H. Kogelnick, and R. Kompfner, Appl. Opt. 3, 523 (1964).

5. D. B. Oh, M. E. Paige, and D. S. Bomse, Appl. Opt. 37, 2499 (1998).

6. P. C. D. Hobbs, "Reaching the shot noise limit for $\$ 10$," Optics \& Photonics News (April, 1991), p. 17.

7. L. S. Rothman, et al., J. Quant. Spectrosc. Radiat. Transfer 48, 469 (1992).

8. H. Sasada, J. Chem. Phys. 88, 767 (1988). 